\title{
Hadron-Hadron Scattering at High Energies
}

\author{
Erasmo Ferreira \\ Instituto de Física, Universidade Federal do Rio de Janeiro \\ Rio de Janeiro 21945-970, RJ, Brazil
}

Received 7 January, 2000

\begin{abstract}
We review the role of the QCD vacuum structure in the determination of the properties of states and processes occurring in the confinement regime of QCD. The vacuum correlation model of nonperturbative QCD is mentioned as a bridge between the fundamental theory and the description of the experiments. The model of the stochastic vacuum provides the framework in which a simple and effective description of the high-energy $p p$ and $\bar{p} p$ data can be given, leading to a determination of relevant parameters of non-perturbative QCD and to a good description of the data. A slow increase of the hadronic radii with the energy accounts for the energy dependence of all observables.
\end{abstract}

\section{Physical QCD Vacuum and High-Energy Phenomenology}

A first observed manifestation of vacuum properties as a source of strong interaction dynamics occurred in the Regge phenomenology of high-energy elastic processes. A dominating and universal contribution to these processes consists in the exchange of an entity, called pomeron, carrying the quantum numbers of the vacuum.

The general features of the hadronic elastic processes $(p p, \bar{p} p, \pi p, K p, \ldots)$ at high energies are rather simple to describe [1]. For all processes, there is a strong forward peak, with the elastic differential crosssection $d \sigma^{e \ell} / d t$ ( $t$ is the squared momentum transfer four-vector) decreasing exponentially with $t$. The total cross-sections first decrease with the energy, until a minimum is reached at an energy around $10 \mathrm{GeV}$, and then increase again, slowly. The values of the total cross-section $\sigma^{T}(s)$ and of the slope parameter of the elastic differential cross-section

$$
B=\left.\frac{d}{d t}\left(\ln \frac{d \sigma^{e \ell}}{d t}\right)\right|_{t=0},
$$

are the basic characteristic quantities of these hadronic elastic processes at very low momentum transfers. These quantities are well described through the Regge exchange phenomenology, developed since the years 1960's. Actually, the Regge pole parametrization [2] yields an excellent phenomenological representation of the bulk of the data on high-energy scattering at small momentum transfers, $t \lesssim 1 \mathrm{GeV}^{2}$. The total crosssection can be written in this approach as

$$
\sigma^{T}=2 \sum_{i} \beta_{i}^{2}(0)\left(\frac{s}{s_{0}}\right)^{\alpha_{i}(0)-1},
$$

and the differential elastic cross-section as

$$
\frac{d \sigma^{e \ell}}{d t}=\frac{1}{4 \pi}\left[\sum_{i} \beta_{i}^{2}(t)\left(\frac{s}{s_{0}}\right)^{\alpha_{i}(t)-1}\right]^{2},
$$

each term of the sums corresponding to a Regge trajectory. At high energies, the process is dominated by the term with the largest value of $\alpha_{i}(0)$, the so-called pomeron trajectory, $\alpha_{1}(t) \equiv \alpha_{p}(t)$, with the quantum numbers of the vacuum $(J=0, I=0, C=+1)$. It has been shown by Donnachie and Landshoff [3] that an excellent description of the scattering data at high energies and small momentum transfers can be obtained with the exchange of one pomeron, with a linearly increasing Regge trajectory $\alpha_{p}(t)=1.0808+0.25 t$. The parameter $\beta_{p}$ determines the strength of the pomeron coupling to the hadrons. For higher momentum transfers, terms corresponding to two and more pomeron exchanges must be added to the amplitude. The value $\alpha_{p}(0)=1.0808$, being larger than 1 , would lead to a violation of the Froissart-Martin bound [4] for extremely high values of $s$, and there it must be modified by the presence of Regge cuts, which occur naturally in a Reggeon field theory.

With the pomeron trajectory alone contributing to the Regge expansions in eqs.(2),(3), we obtain for the energy dependence of the total cross-section

$$
\sigma^{T}(s)=\sigma^{T}\left(s_{0}\right)\left(s / s_{0}\right)^{0.0808},
$$

and of the slope parameter

$$
B(s)-B\left(s_{0}\right)=2 \alpha^{\prime}(t) \log \left(s / s_{0}\right),
$$

where the $\mathrm{t}$ dependence of the residue $\beta(t)$ has been neglected. A direct relation between the values of total 
cross-sections and slope parameters is

$$
\sigma^{T}(s)=\sigma^{T}\left(s_{0}\right) \exp \left[\frac{0.0808}{2 \alpha^{\prime}(t)}\left[B(s)-B\left(s_{0}\right)\right]\right] .
$$

All these relations (4)-(6) are well fulfilled by the data at high energies.

Quantum chromodynamics, as the fundamental theory of the strong interactions, should be able to explain this successful and simple Regge phenomenology. Since the pomeron has the quantum numbers of the vacuum, it is natural to associate its exchange with the exchange of gluons. The understanding of the detailed nature of the gluon-field processes behind this phenomenology is of course an important problem. It is now understood that many important features are due to non-perturbative QCD effects. Soft processes, respecting (even microscopically) the quark and colour confinement in the colliding hadrons, is a domain where the non-perturbative aspects of QCD can be explored and studied. This domain mixes the parameters describing properties of the QCD field (gluon condensate, correlation length) with those describing the colourless hadrons. The effective dynamics providing the basis for the phenomenological description of the data must have the characteristic features of the pomeron exchange mechanism of Regge phenomenology [2]: vacuum quantum numbers exchanged between well determined and unchanged hadronic structures. This mechanism leads, for all hadronic systems, to total cross-sections which increase with the energy [3] somewhat like $s^{0.0808}$.

Since at small momentum transfers the strong coupling constant becomes large, one has to rely either on refined resummation schemes in perturbation theory or on non-perturbative models. Landshoff and Nachtmann [5] have constructed a model in which the pomeron is described by the exchange of two gluons with modified propagators, containing a new length scale $\Lambda$, which implies a modification of the long-range QCD forces. Nachtmann later refined this model [6], describing a system of two quarks interacting through an external vector field (gluon field), which is supposed to vary slowly in time, compared with the frequency associated to high-energy quark motion. Since the quarks in the problem have very high energies, and only very small angle scattering is considered, the WKB (eikonal) approximation for the scattering in an external field can be used, and the quarks can be put in light-like paths.

Hadron-hadron scattering has been treated in the same framework [7], with the purpose of explaining the elastic scattering data in a non-perturbative QCD framework. This treatment requires the functional integration over the external gluon fields (denoted by the bracket $\rangle_{A}$ ), which cannot be performed exactly. Use is then made of the same vacuum correlation model $[8,9]$ introduced in Euclidean field theory for investigations of hadron spectroscopy, where it provides an explanation of confinement (the linearly rising potential) [10] as a dynamical consequence of the vacuum structure. The basic assumption of the model is that the complicated integration over the low-frequency (non-perturbative) contributions to the gluon fields can be approximated by a cluster expansion, ideally by a Gaussian process, which is determined by the correlators of two fields.

The hadronic structure enters the calculation in the simplest way, through Gaussian wave-functions, with a radial parameter $\mathrm{S}$, describing the sizes of the particles. Mesons are treated as simple $q \bar{q}$ systems. Baryons have been treated either through a configuration of three quarks symmetrically distributed in space or through a diquark model (in this case the baryons enter the calculation in a form totally similar to that of the mesons). At the end, hadron physics enters in the results for the observables in high-energy scattering only through the hadronic size parameters S. The relevant QCD parameters in the calculation are the gluon condensate $\left\langle g^{2} F F\right\rangle$ and the correlation length $a$.

The evaluation of the hadron-hadron scattering amplitudes proceeds through the evaluation of eikonal functions, and of averages over the hadronic wavefunctions. After the necessary trace evaluations (the hadrons are colour-singlet objects) and numerical integrations, the profile functions that give a representation for the amplitudes in impact parameter space are otained. Then the expressions for the observables of total cross-section and logarithmic slope are constructed [7], combining QCD quantities and hadron extension parameters in the forms

$$
\sigma^{T}=\alpha\left(\frac{S_{1} S_{2}}{a^{2}}\right)^{\beta / 2}\left(\kappa\left\langle g^{2} F F\right\rangle\right)^{2} a^{10},
$$

and

$$
B=1.858 a^{2}+\frac{\gamma}{2}\left(S_{1}^{2}+S_{2}^{2}\right),
$$

where $S_{1}$ and $S_{2}$ denote the hadron sizes.

Comparing the above expressions with those of Regge phenomenology, eqs.(4) and (5), we observe that now the parameters $S_{1}, S_{2}$ representing the hadronic extensions have taken the place of the energy parameter $s$. If we consider hadron-hadron scattering for hadrons of equal sizes (as in $p p$ and $p \bar{p}$ scattering), a direct relation between the observables $\sigma^{T}$ and $B$, analogous to eq.(6), can be obtained by eliminating $S=S_{1}=S_{2}$, and it has the form

$$
\begin{aligned}
\sigma_{\text {pom }}^{T}=\alpha \gamma^{-\beta / 2}(\kappa< & \left.g^{2} F F>\right)^{2} a^{(10-2 \beta / 2)} \\
& \times\left(B-1.858 a^{2}\right)^{\beta / 2} .
\end{aligned}
$$

It is very interesting and important that both eqs.(6) and (9) represent well the present data, which go up to the energy of $1800 \mathrm{GeV}$.

Using the experimental data for $\sigma^{T}$ and $B$ at a given energy, eqs.(7) and (8) provide a relation between values of the gluon condensate and the correlation length. Independent relations between these two 
quantities can be extracted from the lattice calculation [11], and also from the results of the vacuum correlation model derivation for the linear quark-quark potential $[9,10]$. These three independent relations fit together perfectly well, providing a unique determination of the values of $\left\langle g^{2} F F\right\rangle$ and $a$, which are two fundamental properties of the physical vacuum of quantum chromodynamics.

Several models relate the total high-energy crosssections to the hadronic radii [12]. This is a characteristic feature also of the model of the stochastic vacuum which gives specific predictions for the size dependence of the high-energy observables for different hadronic systems [7]. These predictions account for the observed ratios of $\pi p$ to $p p$ (or $\bar{p} p$ ) total cross-sections, which have been thought of as indications supporting additive quark models, and also account for the important flavour dependence of the observables. The model of the stochastic vacuum treats simultaneously the pp and $\bar{p} p$ systems, describing the high-energy data in terms of non-perturbative QCD parameters, and relating the energy dependence of the observables with radius dependence. The knowledge of the hadronic structures required for the description of the soft high-energy data does not go beyond the information on their sizes, the simplest and most trivial transverse wave-function giving all information required for the determination of the observables. We show that the energy dependence of the total cross-section and of the forward slope parameter can both be accounted for by a slow variation of the radius associated to the transverse wave-function.

The treatment of soft hadron-hadron scattering, essentially including the confinement properties of quantum chromodynamics, cannot be made straightforwardly, requiring use of approximations and models. The model of the stochastic vacuum, originally conceived to treat non-perturbative effects in low-energy hadron physics [8], was later applied to explain highenergy soft scattering [7]. The treatment is based on the concept of loop-loop scattering, which allows a gaugeindependent formulation for the amplitudes. The loops, formed by the quark and antiquark light-like paths in a moving hadron, have their contributions added incoherently, with their sizes weighed by transverse hadronic wave-functions.

We review the results of a more complete calculation of the high-energy observables (total cross-section and slope parameter), in which both Abelian and nonAbelian contributions to the field correlator are taken into account. The role of the parameter $\kappa$ measuring the strength of the non-Abelian part, which was determined in lattice calculations to be about $3 / 4$, is studied and we observe that the range of values that suits the description of the high-energy data leads to a confirmation of the lattice results. We take into account all available data on total cross-sections and slope parameters in $\mathrm{pp}$ and $\overline{\mathrm{p}} \mathrm{p}$ scattering, which consist mainly $[13,14]$ of ISR (CERN) measurements at energies ranging from $\sqrt{s}=23 \mathrm{GeV}$ to $\sqrt{s}=63 \mathrm{GeV}$, of the $\sqrt{s}=541-546$ $\mathrm{GeV}$ measurements in CERN SPS and in Fermilab, and of the $\sqrt{s}=1800 \mathrm{GeV}$ information from the E710 Fermilab experiment. These data are shown in table 1. Besides these, there is a measurement [15] of $\sigma^{T}=65.3 \pm 2.3 \mathrm{mb}$ at $\sqrt{s}=900 \mathrm{GeV}$ and there are the measurements of $\sigma^{T}=80.6 \pm 2.3$ and $B=17.0 \pm 0.25$ $\mathrm{GeV}^{-2}$ in Fermilab CDF [16] at $\sqrt{s}=1800 \mathrm{GeV}$ which seem discrepant with the E-710 experiment at the same energy. A measurement by Burq et al.[17] at $\sqrt{s}=19$ $\mathrm{GeV}$ seems to disagree with the ISR data, presenting a too high value for $B=12.47 \pm 0.10 \mathrm{GeV}^{-2}$ (possibly because the measurements are taken at rather large momentum transfers; for our purposes these should be smaller than the hadronic scale of $\approx 1 \mathrm{GeV}$ ). This point at $\sqrt{s}=19 \mathrm{GeV}$ was taken as the sole input in the first calculation made [7].

Table 1. Experimental high-energy data from CERN ISR, CERN SPS and Fermilab.

\begin{tabular}{ccccc}
\hline & $\begin{array}{c}\sqrt{s} \\
(\mathrm{GeV})\end{array}$ & $\begin{array}{c}\sigma^{T} \\
(\mathrm{mb})\end{array}$ & $\begin{array}{c}B \\
\left(\mathrm{GeV}^{-2}\right)\end{array}$ & $\begin{array}{c}\text { Ref. } \\
{[13]}\end{array}$ \\
\hline \multirow{4}{*}{$p p$} & 23.5 & $39.65 \pm 0.22$ & $11.80 \pm 0.30$ & $(a)$ \\
& 30.6 & $40.11 \pm 0.17$ & $12.20 \pm 0.30$ & $(a)$ \\
& 45.0 & $41.79 \pm 0.16$ & $12.80 \pm 0.20$ & $(b)$ \\
& 52.8 & $42.38 \pm 0.15$ & $12.87 \pm 0.14$ & $(a)$ \\
& 62.3 & $43.55 \pm 0.31$ & $13.02 \pm 0.27$ & $(a)$ \\
\hline & & & & \\
$\bar{p} p$ & 30.4 & $42.13 \pm 0.57$ & $12.70 \pm 0.50$ & $(a)$ \\
& 52.6 & $43.32 \pm 0.34$ & $13.03 \pm 0.52$ & $(a)$ \\
& 62.3 & $44.12 \pm 0.39$ & $13.47 \pm 0.52$ & $(a)$ \\
& 541 & $62.20 \pm 1.50$ & $15.52 \pm 0.07$ & $(c)$ \\
& 546 & $61.90 \pm 1.50$ & $15.28 \pm 0.58$ & $(d)$ \\
& 1800 & $72.20 \pm 2.70$ & $16.72 \pm 0.44$ & $(e)$ \\
\hline
\end{tabular}

In Fig.1 we plot the two observables, $\sigma^{T}$ and $B$ against each other. At the ISR energies we use $\sigma_{\text {pom }}^{T}=(21.70 \mathrm{mb}) s^{0.0808}$ as representative of the non-perturbative contributions, instead of the full experimental values. At the highest energies (541-1800 $\mathrm{GeV}$ ) it is believed that the process is essentially nonperturbative. The relation between the two observables is parametrized in the form

$$
B=B_{0}+C\left(\sigma^{T}\right)^{\Delta},
$$

with $B_{0}=5.38 \mathrm{GeV}^{-2}, C=0.458 \mathrm{GeV}^{-2}, \Delta=0.75$, and with $\sigma^{T}$ given in milibarns. This form is suggested by the results of the calculations with the model of the stochastic vacuum [7], where an interpretation for the meaning of the parameters is given in terms of QCD and hadronic quantities. This is explained in detail later.

In the next section we recall the principles of the evaluation of the observables of high-energy scattering 
in the model of the stochastic vacuum [7]. In the sections that follow we present our new calculations and results.

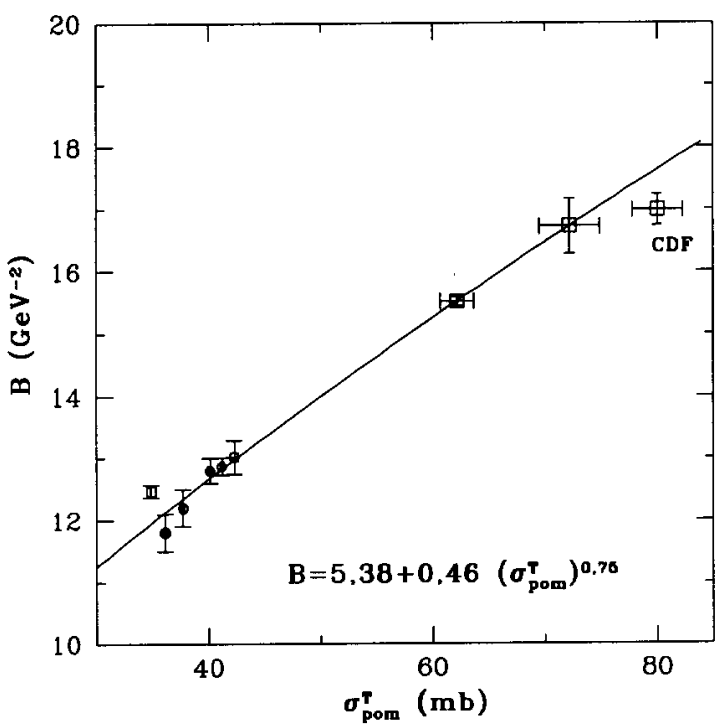

Figure 1. Relation between the two experimental quantities of the $\mathrm{pp}$ and $\overline{\mathrm{p}} \mathrm{p}$ systems. The values of $\sigma^{T}$ at energies up to $62.3 \mathrm{GeV}$ shown in this figure are the $\sigma_{\text {pom values }}^{T}$ as given by the parameterization of Donnachie-Landshoff, namely $\sigma_{\text {pom }}^{T}=(21.70 \mathrm{mb}) s^{0.0808}$. We also included the point [17] at $19 \mathrm{GeV}$ and the Fermilab CDF values [16] at $1800 \mathrm{GeV}$. The values of B for the pp system are shown with circles, while the values for $\bar{p} p$ are represented by squares. The solid line represents eq.(10), with values for $\Delta, B_{0}$ and $\mathrm{C}$ given in the figure.

\section{Nonperturbative QCD and the Model of the Stochastic Vacuum in Soft High Energy Scattering}

The non-perturbative vacuum expectation values (such as gluon condensates) that were first introduced in calculations of hadron spectroscopy [18] were shown by Nachtmann [6] to have fundamental role in soft highenergy scattering. The application of the model of the stochastic vacuum to this problem follows his general analysis, adopting however a different fundamental ingredient. Instead of reducing the hadron-hadron amplitude to quark-quark scattering amplitudes, the basic entities used are scattering amplitudes for Wilson loops in Minkowski space-time. The loops are formed by the trajectories of the quark and the antiquark of the hadronic system, and this approach has the important advantage that the amplitudes are gauge invariant.

The model of the stochastic vacuum [8] is based on the assumption that the low frequency contributions in the functional integral can be taken into account by a simple stochastic process with a converging cluster expansion [19]. The integration is specified by a simple correlator, which is determined by two scales: the strength of the correlator (the value of the gluon condensate) and the correlation length. This simple model leads to confinement in a non-Abelian gauge theory, with a linear potential between static quarks which agrees with phenomenological determinations [20].

In order to guarantee gauge invariance, the model deals with the correlator of the field-strengths $F_{\mu \nu}$, rather than with the expectation values of gauge potentials $A_{\mu}(x)$. In order to give a well defined meaning to the correlator, which is a bilocal object, the colourcontent of all fields must be parallel-transported to a single reference point $w$. Then the parallel-transported field strength tensors

$$
\mathbf{F}_{\mu \nu}(x ; w):=\phi^{-1}(x, w) \mathbf{F}_{\mu \nu}(x) \phi(x, w),
$$

where $\phi(x, w)$ is a non-Abelian Schwinger string from point $w$ to point $x$, must be constructed. This quantity follows the gauge transformation at the fixed reference point $w$

$$
\mathbf{F}_{\mu \nu}(x, w) \rightarrow \mathbf{U}(w) \mathbf{F}_{\mu \nu}(x ; w) \mathbf{U}^{-1}(w),
$$

so that the vacuum expectation value $\left\langle\mathbf{F}_{\mu \nu}(x, w) \mathbf{F}_{\delta \sigma}(y, w)\right\rangle_{A}$ with respect to the low frequencies is a gauge invariant quantity.

With the approximation that the correlator is independent of the reference point $w$, depending only on the difference $z=x-y$, its most general form [8] is given by

$$
\begin{aligned}
& \left\langle\quad g^{2} F_{\mu \nu}^{C}(x, w) F_{\rho \sigma}^{D}(y, w)\right\rangle_{A}=\frac{\delta^{C D}}{8} \frac{1}{12}\left\langle g^{2} F F\right\rangle \\
& \cdot \quad\left\{\kappa\left(\delta_{\mu \rho} \delta_{\nu \sigma}-\delta_{\mu \sigma} \delta_{\nu \rho}\right) \cdot D\left(z^{2} / a^{2}\right)\right. \\
& +\quad(1-\kappa) \cdot \frac{1}{2}\left[\frac{\partial}{\partial z_{\mu}}\left(z_{\rho} \delta_{\nu \sigma}-z_{\sigma} \delta_{\nu \rho}\right)\right. \\
& \left.\left.+\quad \frac{\partial}{\partial z_{\nu}}\left(z_{\sigma} \delta_{\mu \rho}-z_{\rho} \delta_{\mu \sigma}\right)\right] D_{1}\left(z^{2} / a^{2}\right)\right\} .
\end{aligned}
$$

Here $a$ is a characteristic correlation length, $\left\langle g^{2} F F\right\rangle$ is the gluon condensate

$$
\left\langle g^{2} F F\right\rangle=\left\langle g^{2} F_{\mu \nu}^{C}(0) F_{\mu \nu}^{C}(0)\right\rangle_{A},
$$

$C, D=1, \ldots, 8$ are colour indices, and the numerical factors in eq.(13) are chosen in such a way that

$$
D(0)=D_{1}(0)=1 .
$$

Lattice studies [11] show that the ratio $\kappa /(1-\kappa)$ is rather large (about 3$)$, so that $D\left(z^{2} / a^{2}\right)$ gives the dominant contribution. This dominance was the reason for the previous [7] neglect of the contributions from the part $(1-\kappa) D_{1}\left(z^{2} / a^{2}\right)$, which are taken into consideration in the present work. 
The correlator in eq.(13) is the starting point for the evaluation of observables in soft high-energy scattering. In the analysis made by Nachtmann [6], the quark-quark scattering amplitude for the interaction of the quarks with the gluon field is evaluated using the eikonal approximation. If the energy of the quark is very high and the background field has only a limited frequency range, the quark moves on an approximately straight light-like line and the eikonal approximation can be applied. In the limit of high energies there is helicity conservation and spin degrees of freedom can be ignored. This quark-quark scattering amplitude is explicitly gauge dependent. However, we can make use of the fact that in meson-meson scattering for each quark there is an antiquark moving on a nearly parallel line. The meson must be a colour singlet state under local gauge transformations, and to construct such a colourless state we have to parallel-transport the colour content from the quark to the antiquark. Since this parallel-transport of the colours is made by a Schwinger string, we obtain for the meson a rectangular Wilson loop whose light-like sides are formed by the quark and antiquark paths, and whose front ends are the Schwinger strings. The direction of the path of an antiquark is effectively the opposite of that of a quark, so that the loop has a well defined internal direction.

The resulting loop-loop amplitude is then specified, not only by the impact parameter, but also by the transverse extension vectors $\vec{R}_{1}$ and $\vec{R}_{2}$. In the transverse plane the two interacting loops are seen as shown in Fig.2.

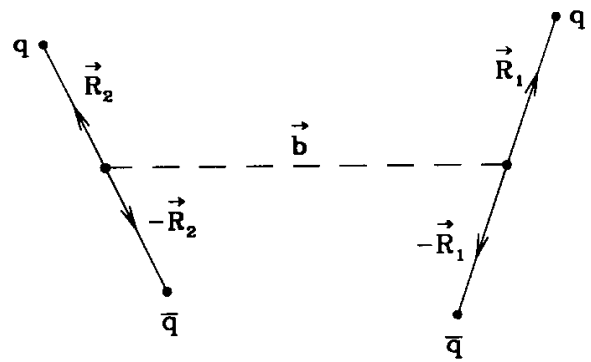

Figure 2. View in the transverse plane of the two loops that represent the paths of quark and antiquark in meson-meson scattering. The vectors $\vec{R}_{1}$ and $\vec{R}_{2}$ represent components of the meson transverse wave-functions. The vector $\vec{b}$ is the impact parameter vector connecting the geometric center of the two hadrons.

The functional integration over $A$ is evaluated using the model of the stochastic vacuum. Since the correlator is given in terms of the parallel-transported field tensor $\mathbf{F}_{\mu \nu}(x, w)$, the line integrals $\int \mathbf{A}_{\mu} d z^{\mu}$ are transformed into surface integrals over the field tensor with the help of the non-Abelian Stokes-theorem. The integrations are then extended over open surfaces $\mathrm{S}_{1}$ and $\mathrm{S}_{2}$ having the loops $\mathrm{L}_{1}$ and $\mathrm{L}_{2}$ as contours.

The exponential being expanded, the expectation value can be calculated assuming factorization in a
Gaussian process. In the expansion of the trace of the exponential at least two terms are necessary, because $\operatorname{tr} \tau_{A}=0$, we obtain the lowest order contribution to the loop-loop scattering amplitude. The integration surfaces and details of the calculation have been described before [7]. The higher order terms are shown to be small as compared to the leading term, and can be neglected. In this approximation the surface ordering becomes irrelevant. The expectation values of the product of four fields is evaluated using the factorization hypothesis

$$
\begin{aligned}
\left\langle F^{C_{1}} F^{C_{2}} F^{D_{1}} F^{D_{2}}\right\rangle & =\left\langle F^{C_{1}} F^{C_{2}}\right\rangle\left\langle F^{D_{1}} F^{D_{2}}\right\rangle \\
+\left\langle F^{C_{1}} F^{D_{1}}\right\rangle\left\langle F^{C_{2}} F^{D_{2}}\right\rangle & \left.+\left\langle F^{C_{1}} F^{D_{2}}\right\rangle\left\langle F^{C_{2}} F^{D_{1}}\right\rangle 16\right)
\end{aligned}
$$

It is convenient to introduce the eikonal function $\chi$ in terms of which the loop-loop amplitude $J\left(\vec{b}, \vec{R}_{1}, \vec{R}_{2}\right)$ is given to the lowest order in the correlator by

$$
J\left(\vec{b}, \vec{R}_{1}, \vec{R}_{2}\right)=-\frac{1}{576}\left[\chi\left(\vec{b}, \vec{R}_{1}, \vec{R}_{2}\right)\right]^{2} .
$$

In order to extract as a factor the value of the gluon condensate, it is useful to introduce a reduced eikonal function and a reduced loop-loop scattering amplitude through

$$
\widetilde{\chi}\left(\vec{b}, \vec{R}_{1}, \vec{R}_{2}\right) \equiv \frac{12}{\left\langle g^{2} F F\right\rangle} \chi\left(\vec{b}, \vec{R}_{1}, \vec{R}_{2}\right)
$$

and

$$
\begin{aligned}
& \widetilde{J}_{L L^{\prime}}\left(\vec{b}, \vec{R}_{1}, \vec{R}_{2}\right) \equiv \frac{1}{\left[\left\langle g^{2} F F\right\rangle\right]^{2}} J_{L L^{\prime}}\left(\vec{b}, \vec{R}_{1}, \vec{R}_{2}\right) \\
& =-\frac{\left[\widetilde{\chi}\left(\vec{b}, \vec{R}_{1}, \vec{R}_{2}\right)\right]^{2}}{144 \cdot 576}
\end{aligned}
$$

We have introduced the indices $L, L^{\prime}$ to indicate the two loops.

To be applied to high-energy scattering, the model of the stochastic vacuum must be translated from Euclidean space-time, to the Minkowski continuum. The correlation functions $D\left(z^{2} / a^{2}\right)$ and $D_{1}\left(z^{2} / a^{2}\right)$ must fall off for negative $z^{2}$ values (corresponding to Euclidean distances), and must have well defined Fourier transforms in the Minkowski metric, since these enter in the scattering amplitudes.

The loop-loop eikonal function is determined by the geometry of the two loops and by the form of the correlation functions. In eq.(13) there appear two independent arbitrary scalar functions, $D\left(z^{2} / a^{2}\right)$ and $D_{1}\left(z^{2} / a^{2}\right)$, which are supposed to fall off at large distances with characteristic lengths $a$, called correlation lengths. Lattice calculations [11] show however that the forms of $D$ and $D_{1}$ in the Euclidean region at large distances are similar (exponential decreases with same 
rates), with the contribution from the term with $D$ in the correlator being about 3 times larger than that from $D_{1}$. We then adopt the same shapes $D \equiv D_{1}$, and $\kappa=3 / 4$.

A convenient general form [7] for the correlation function is

$$
\begin{gathered}
D^{(n)}\left(-|\vec{\xi}|^{2}\right)=\frac{1}{2^{n-3} \Gamma(n-3)}\left(\rho_{n}|\vec{\xi}|\right)^{n-3} \\
{\left[(n-1) K_{n-3}\left(\rho_{n}|\vec{\xi}|\right)-\frac{1}{2}\left(\rho_{n}|\vec{\xi}|\right) K_{n-2}\left(\rho_{n}|\vec{\xi}|\right)\right],}
\end{gathered}
$$

where $K_{\nu}(x)$ is the modified Bessel function, $n \geq 4$, and

$$
\rho_{n}=\frac{3 \sqrt{\pi}}{4} \frac{\Gamma(n-5 / 2)}{\Gamma(n-3)} .
$$

The dependence of the final results on the particular choice for $\mathrm{n}$ is not very marked, the reason being that all correlation functions are normalized to 1 at the origin, and decrease exponentially at large distances. It is enough that the chosen function falls monotonically and smoothly in the range of physical influence (up to about one fermi, say), and there cannot be much difference in the results obtained using different reasonable analytical forms. The simpler choice is $n=4$, which in the Euclidean region leads to a good representation of the lattice calculations [11]. We then have for the correlation function

$D^{(4)}\left(-|\vec{\xi}|^{2}\right)=\left(\rho_{4}|\vec{\xi}|\right)\left[K_{1}\left(\rho_{4}|\vec{\xi}|\right)-\frac{1}{4}\left(\rho_{4}|\vec{\xi}|\right) K_{0}\left(\rho_{4}|\vec{\xi}|\right)\right]$

with

$$
\rho_{4}=\frac{3 \pi}{8} .
$$

In the evaluation of the (Euclidean) Wilson loop in the model of the stochastic vacuum the $\mathrm{D}$ part of the correlator leads [8] to the area law for a Wilson loop, and to a relation involving the condensate $\kappa\left\langle g^{2} F F\right\rangle$, the correlation length $a$ and the string tension $\rho$

$$
\rho=\frac{\kappa \pi}{144}\left\langle g^{2} F F\right\rangle a^{2} \int_{0}^{\infty} D\left(-u^{2}\right) d u^{2} .
$$

For the family of correlators written above the integration can be performed analytically and for the case $n=4$ the result gives

$$
\kappa\left\langle g^{2} F F\right\rangle=\frac{81 \pi}{8 a^{2}} \rho .
$$

We thus say that $D$ represents the confining correlator, while $D_{1}$ is the non-confining (and Abelian) part.

After the limits are taken, which make the long sides of the rectangular Wilson loops tend to $\pm \infty$ in the direction of the colliding beams, the remaining variables in the integrands are coordinates of points in the transverse plane. The distances $z$ between such points enter in the final expressions for the eikonal functions $\chi$ as arguments of the two-dimensional inverse Fourier transform, which is given by

$$
\begin{array}{r}
\mathcal{F}_{2}^{(4)}\left(-|\vec{\xi}|^{2}\right)=\frac{32}{9 \pi}\left(\rho_{4}|\vec{\xi}|\right)^{2}\left[2 K_{0}\left(\rho_{4}|\vec{\xi}|\right)\right. \\
\left.-\left(\frac{4}{\rho_{4}|\vec{\xi}|}-\rho_{4}|\vec{\xi}|\right) K_{1}\left(\rho_{4}|\vec{\xi}|\right)\right] \\
=-\frac{32}{9 \pi} \Delta_{2}\left[\left(\rho_{4}|\vec{\xi}|\right)^{3} K_{3}\left(\rho_{4}|\vec{\xi}|\right)\right]
\end{array}
$$

where $\Delta_{2}$ is the 2-dimensional Laplacian operator, and $\vec{\xi}$ is any two-dimensional vector of the transverse plane and $K_{3}$ is a modified Bessel function. This Laplacian form is important in the calculation, as it allows lowering the order of the integrations, through Gauss theorem.

\section{Profile Function for Hadron-Hadron Scattering}

We now introduce the notation $\vec{R}(I, J)$, where the first index $(I=1,2)$ specifies the loop, and the second specifies the particular quark or antiquark $(\mathrm{J}=1$ or 2$)$ in that loop.

Fig.3 shows a projection on the transverse scattering plane. The vectors $\vec{Q}(K, L)$ in the transverse plane connect the reference point $C$ (with coordinates $w$ ) to the positions of the quarks and antiquarks of the loops 1 and 2. The quantity $\psi(K, L)$ is the angle between $\vec{Q}(1, K)$ and $\vec{Q}(2, L)$.

In the evaluation of the eikonal functions $\chi(\vec{b}, \vec{R}(1,1), \vec{R}(2,1))$ coming from the confining case a typical resulting contribution is

$$
\begin{array}{r}
\int_{0}^{1} d \alpha \int_{0}^{1} d \beta \cos \Psi(1,1) \\
\mathcal{F}_{2}^{(4)}\left(-|\alpha \vec{Q}(1,1)-\beta \vec{Q}(2,1)|^{2}\right),
\end{array}
$$

where $\mathcal{F}_{2}^{(4)}$ is the above mentioned two-dimensional Fourier transform of the correlator with $n=4$. Taking advantage of the Laplacian form, we can apply Gauss' theorem in two dimensions and eliminate one further integration. The term from the non-confining correlator has a total derivative under the integration sign, and in this part one more integration can be immediately made. 


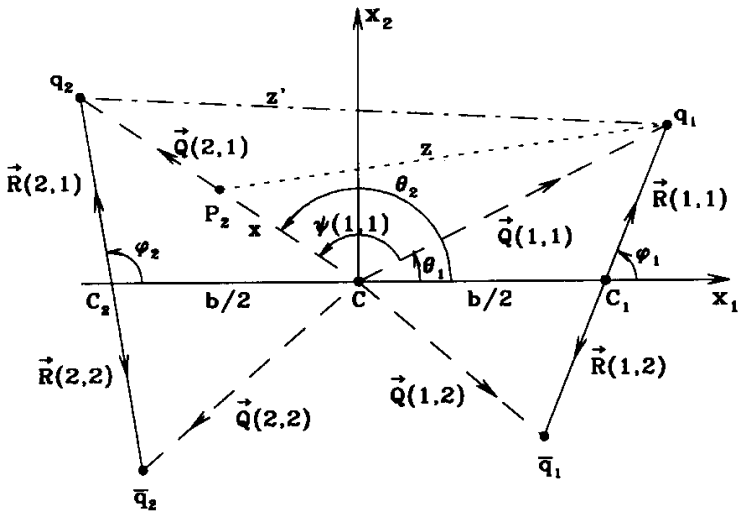

Figure 3. Geometrical variables of the transverse plane, which enter in the calculation of the eikonal function for meson-meson scattering. The points $C_{1}$ and $C_{2}$ are the meson centers. In the integration, $P_{2}$ runs along the vector $\vec{Q}(2,1)$, changing the length $z$, which is the argument of the characteristic correlator function. In analogous terms, points $P_{1}, \bar{P}_{1}$ and $\bar{P}_{2}$ run along $\vec{Q}(1,1), \vec{Q}(1,2)$ and $\vec{Q}(2,2)$. This explains the four terms that appear inside the brackets multiplying $\kappa$ in the expression for the loop-loop amplitude. The length $z^{\prime}$ of the dot-dashed line is the argument of the Bessel function arising from the non-confining correlator $D_{1}$; there are four such terms, appearing inside the brackets multiplying $(1-\kappa)$.

We then write for the eikonal function of the looploop amplitude

$$
\begin{array}{ll}
\tilde{\chi} & (\vec{b}, \vec{R}(1,1), \vec{R}(2,1))=\kappa[ \\
- & \cos \psi(1,1) I[Q(1,1), Q(2,1), \psi(1,1)] \\
- & \cos \psi(2,2) I[Q(1,2), Q(2,2), \psi(2,2)] \\
+ & \cos \psi(1,2) I[Q(1,1), Q(2,2), \psi(1,2)] \\
+ & \cos \psi(2,1) I[Q(1,2), Q(2,1), \psi(2,1)]] \\
+ & (1-\kappa)[-W[Q(1,1), Q(2,1), \psi(1,1)] \\
- & W[Q(1,2), Q(2,2), \psi(2,2)] \\
+ & W[Q(1,1), Q(2,2), \psi(1,2)] \\
+ & W[Q(1,2), Q(2,1), \psi(2,1)]] .
\end{array}
$$

The quantities $I$ which represent the non-Abelian contributions are given by integrations along the dashed lines of the figure:

$$
\begin{aligned}
I[Q(1, K), Q(2, L), & \psi(K, L)]=\frac{32}{9 \pi}\left(\frac{3 \pi}{8}\right)^{2} \\
& \times\left\{Q(1, K) \int_{0}^{Q(2, L)}\left[Z_{1}(x)\right]\right. \\
& \times K_{2}\left[\frac{3 \pi}{8} \sqrt{Z_{1}(x)}\right] d x \\
+ & Q(2, L) \int_{0}^{Q(1, K)}\left[Z_{2}(x)\right] \\
& \left.\times K_{2}\left[\frac{3 \pi}{8} \sqrt{Z_{2}(x)}\right] d x\right\},
\end{aligned}
$$

with $Q(K, L)=|\vec{Q}(K, L)|$ and where

$$
\begin{aligned}
& Z_{1}(x)=Q(1, K)^{2}+x^{2}-2 x Q(1, K) \cos \psi(K, L) \\
& Z_{2}(x)=Q(2, L)^{2}+x^{2}-2 x Q(2, L) \cos \psi(K, L)
\end{aligned}
$$

The quantities $\mathrm{W}$, which come from the non-confining part of the correlator, are given by

$$
\begin{gathered}
W[Q(1, K), Q(2, L), \psi(K, L)]= \\
\frac{32}{9 \pi} 2 \frac{3 \pi}{8}\left[Z_{3}\right]^{3 / 2} K_{3}\left[\frac{3 \pi}{8} \sqrt{Z_{3}}\right]
\end{gathered}
$$

where

$$
\begin{gathered}
Z_{3}=Q(1, K)^{2}+Q(2, L)^{2} \\
-2 Q(1, K) Q(2, L) \cos \psi(K, L) .
\end{gathered}
$$

From the eikonal function $\widetilde{\chi}$ we construct the looploop amplitude $\widetilde{J}_{L_{1} L_{2}}\left(\vec{b}, \vec{R}_{1}, \vec{R}_{2}\right)$, where $\vec{R}_{1}$ and $\vec{R}_{2}$ are shorthand notations for $\vec{R}(1,1)$ and $\vec{R}(2,1)$ respectively.

The hadron-hadron amplitude is constructed from the loop-loop amplitude using a simple quark model for the hadrons. Since our amplitude is independent of the momentum of the quarks (as long as the energy is high enough to ensure light-like paths), the dependence of the wave-functions on the longitudinal momenta of the quarks can be neglected, and we thus only consider the transverse dependence, which is given by the Fourier transform of the transverse wave-function. We thus obtain the hadron-hadron scattering amplitude by smearing over the values of $\vec{R}_{1}$ and $\vec{R}_{2}$ in eq.(17) with transverse wave-functions $\psi(\vec{R})$.

Taking into account the results of the previous analysis of different hadronic systems [7], in the present calculation we only consider for the proton a diquark structure, where the proton is described as a meson, in which the diquark replaces the antiquark. Thus these expressions apply equally well to meson-meson, mesonbaryon and baryon-baryon scattering.

For the hadron transverse wave-function we make the ansatz of the simple Gaussian form

$$
\psi_{H}(R)=\sqrt{2 / \pi} \frac{1}{S_{H}} \exp \left(-R^{2} / S_{H}^{2}\right),
$$

where $S_{H}$ is a parameter for the hadron size.

We then write the reduced profile function of the eikonal amplitude

$$
\begin{gathered}
\widehat{J}_{H_{1} H_{2}}\left(\vec{b}, S_{1}, S_{2}\right)=\int d^{2} \vec{R}_{1} \int d^{2} \vec{R}_{2} \\
\widetilde{J}_{L_{1} L_{2}}\left(\vec{b}, \vec{R}_{1}, \vec{R}_{2}\right)\left|\psi_{1}\left(\vec{R}_{1}\right)\right|^{2}\left|\psi_{2}\left(\vec{R}_{2}\right)\right|^{2},
\end{gathered}
$$

which is a dimensionless quantity.

For short, from now on we write $J(b)$ or $J(b / a)$ to represent $\widehat{J}\left(\vec{b}, S_{1}, S_{2}\right)$. 
The contributions of both the confining and nonconfining correlators to the eikonal function and to the observables in high-energy scattering are being taken into account. Aiming at the pp and $\bar{p}$ p systems, we only consider the case $S_{1}=S_{2}=S$. Fig. 4 shows a comparison between the results for the profile functions $J(b / a)$ corresponding to $S / a=2.4$ in the cases of pure confining $(\kappa=1)$, pure non-confining $(\kappa=0)$ and mixed $(\kappa=3 / 4)$ correlators, in order to exhibit their differences.

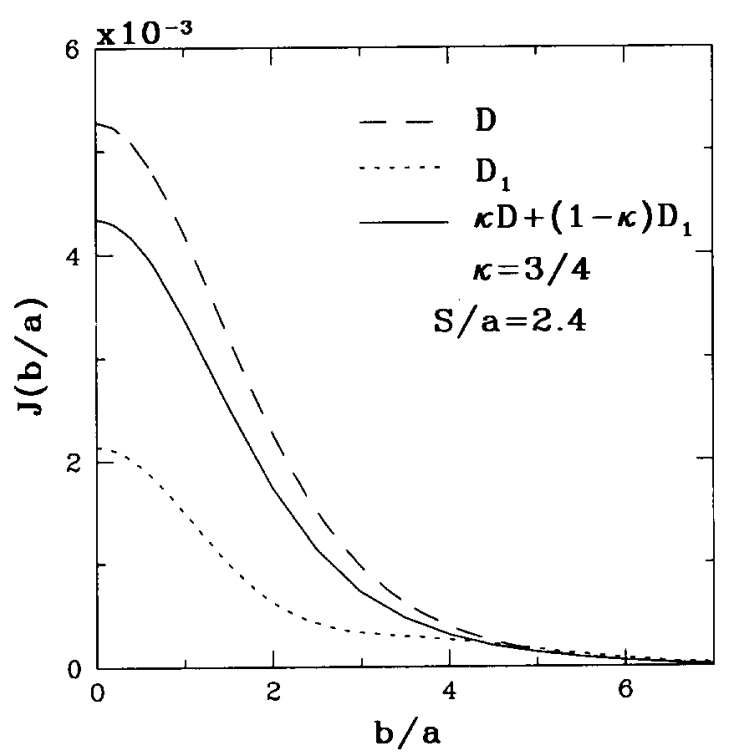

Figure 4. Dimensionless profile functions $J(b / a)$ for $S / a=$ 2.4 obtained in the cases of pure confining $(\kappa=1)$, pure non-confining $(\kappa=0)$ and mixed $(\kappa=3 / 4)$ correlators.

The dimensionless hadron-hadron scattering amplitude in the eikonal approach is given by

$$
\begin{array}{r}
T_{H_{1} H_{2}}=i s\left[\left\langle g^{2} F F\right\rangle a^{4}\right]^{2} a^{2} \\
\int d^{2} \vec{b} \exp (i \vec{q} \cdot \vec{b}) \widehat{J}_{H_{1} H_{2}}\left(\vec{b}, S_{1}, S_{2}\right),
\end{array}
$$

where the impact parameter vector $\vec{b}$ and the hadron sizes $S_{1}, S_{2}$ are in units of the correlation length $a$, and $\vec{q}$ is the momentum transfer projected on the transverse plane, in units of $1 / a$, so that the momentum transfer squared is $t=-|\vec{q}|^{2} / a^{2}$. For convenience, in the expression above we have explicitly factorized the dimensionless combination $\left\langle g^{2} F F\right\rangle a^{4}$. The normalization for $T_{H_{1} H_{2}}$ is such that the total cross-section is obtained through the optical theorem by

$$
\sigma^{T}=\frac{1}{s} \operatorname{Im} T_{H_{1} H_{2}},
$$

and the differential cross-section is given by

$$
\frac{d \sigma^{e \ell}}{d t}=\frac{1}{16 \pi s^{2}}\left|T_{H_{1} H_{2}}\right|^{2} .
$$

To write convenient expressions for the observables, we define the dimensionless moments of the profile function (as before, with $b$ in units of the correlation length a)

$$
I_{k}=\int d^{2} \vec{b} b^{k} \widehat{J}(b), k=0,1,2, \ldots
$$

which depend only on $S / a$, and the Fourier-Bessel transform

$$
I(t)=\int d^{2} \vec{b} J_{0}(b a \sqrt{|t|}) \widehat{J}(b)
$$

where $J_{0}(b a \sqrt{|t|})$ is the zeroth-order Bessel function. Then

$$
T_{H_{1} H_{2}}=i s\left[\left\langle g^{2} F F\right\rangle a^{4}\right]^{2} a^{2} I(t) .
$$

Since $J(b)$ is real, the total cross section $\sigma^{T}$, the slope parameter B (slope at $t=0$ ) and the differential elastic cross-section are given by

$$
\begin{gathered}
\sigma^{T}=I_{0}\left[\left\langle g^{2} F F\right\rangle a^{4}\right]^{2} a^{2} \\
B=\left.\frac{d}{d t}\left(\ln \frac{d \sigma^{e \ell}}{d t}\right)\right|_{t=0}=\frac{1}{2} \frac{I_{2}}{I_{0}} a^{2}=K a^{2},
\end{gathered}
$$

and

$$
\frac{d \sigma^{e \ell}}{d t}=\frac{1}{16 \pi} I(t)^{2}\left[\left\langle g^{2} F F\right\rangle a^{4}\right]^{4} a^{4} .
$$

We have here defined

$$
K=\frac{1}{2} \frac{I_{2}}{I_{0}} .
$$

We observe that in the lowest order of the correlator expansion the slope parameter $B$ does not depend on the value of the gluon condensate $\left\langle g^{2} F F\right\rangle$ and, once the proton radius $S$ is known, may give a direct determination of the correlation length.

The QCD strength and length scale have been factorized in the expressions for the observables, and the correlation length appears as the natural unit of length for the geometric aspects of the interaction. These aspects are contained in the quantities $I_{0}(S / a)$ and $I_{2}(S / a)$, which depend on the hadronic structures and on the shapes and relative weights (parameter $\kappa$ ) of the two correlation functions. It has been shown [7] that for the case $\kappa=1$ the two moments have simple form as functions of $S / a$. To consider arbitrary values for $\kappa$, we remark that the profile function and its moments are quadratic functions of $\kappa$, as they result from integrations of the squares of a (symbolic) combination $\kappa D+(1-\kappa) D_{1}$. The profile function $J(b / a)$ for an arbitrary value of the weight $\kappa$ can be obtained once the profile functions have been determined for three different values of $\kappa$. It is shown in the next section that the moments $I_{0}(S / a)$ and $I_{2}(S / a)$ for arbitrary $\kappa$ (with $0 \leq \kappa \leq 1)$ can be represented by similarly simple expressions.

It is important that the high-energy observables $\sigma^{T}$ and $B$ require only the two low moments $I_{0}, I_{2}$ of the profile functions. The curvature of the forward peak depends on higher moments and on the long distance behavior of $J(b / a)$. 


\section{Experimental Observables and QCD Parameters}

The curves for $I_{0}=\sigma^{T} /\left[\left\langle g^{2} F F\right\rangle^{2} a^{10}\right]$ and $K=B / a^{2}$ can be parameterized as functions of $S / a$ with simple powers, with good accuracy for $0 \leq \kappa \leq 1$. The convenient expressions are

$$
I_{0}=\alpha\left(\frac{S}{a}\right)^{\beta}
$$

and

$$
K=\eta+\gamma\left(\frac{S}{a}\right)^{\delta}
$$

The numerical values of the parameters $\alpha, \beta, \eta, \gamma, \delta$ and the ratio $\delta / \beta$ (which is of particular importance for the comparison with the data) for $\kappa=3 / 4$ are

$$
\begin{gathered}
\alpha=0.6532 \times 10^{-2}, \beta=2.791, \eta=2.030 \\
\gamma=0.3293, \delta=2.126, \delta / \beta=0.762 .
\end{gathered}
$$

The parameterizations of the total cross-section and of the slope parameter $B$ are very convenient for comparison of the results of the model of the stochastic vacuum with experiment. In order to have a wide range of data to extract reliable information on QCD parameters, we concentrate on elastic $p p$ and $p \bar{p}$ scattering. The data extending from the ISR range $(20-60 \mathrm{GeV})$ to the Fermilab energy $(1800 \mathrm{GeV})$ are presented in table 1 and in Fig.1.

The non-perturbative calculation made with the model of the stochastic vacuum corresponds to the phenomenological pomeron exchange of Regge phenomenology [2, 3]. Donnachie and Landshoff [3] found that the parameterization $\sigma_{\text {pom }}^{T}(p p, \bar{p} p)=$ $(21.70 \mathrm{mb}) s^{0.0808}$ (with $\mathrm{s}$ in $\mathrm{GeV}^{2}$ ) works well over a wide range of data above $\sqrt{s}=5 \mathrm{GeV}$, so that we may use this expression to represent the pomeron contribution at the energies where the non-pomeron part is important (the ISR energies). At 541 and $1800 \mathrm{GeV}$ we assume that pomeron exchange dominates the scattering process, and ignore possible differences between pp and $\bar{p}$ p systems. We then take the data at these two highest energies as input, and predict the values for the lower (ISR) energies.

The values of the slope parameter related to the pomeron exchange mechanism are not known, and must be predicted by a model. Our model makes specific predictions for the relation between $\sigma_{\text {pom }}^{T}$ and $\mathrm{B}_{\text {pom }}$, and we need good data to test accurately these predictions. The differences $B(p \bar{p})-B(p p)$ are $0.50,0.16$ and $0.45 \mathrm{GeV}^{-2}$ at $30.5,52.7$ and $62.3 \mathrm{GeV}$ respectively, with error bars typically $\pm 0.55 \mathrm{GeV}^{-2}$ (see table 1); these differences do not shown a decrease with the increasing energy, as expected from pomeron dominance, but the error bars are too large, larger than the quantities themselves. The situation is simpler with the total cross-sections, where at the same three energies the differences $\sigma^{T}(\bar{p} p)-\sigma^{T}(p p)$ are 2.02, 0.94 and 0.57 $\mathrm{mb}$ respectively, decreasing continuously to zero, and with error bars not larger than the values of the differences. Thus, in the range of the ISR experiments, we see the cross-sections converging to the same pomeronexchange values, but not the slopes.

In Fig.1, besides the ISR and higher energy data, we show the point [17] corresponding to $\sqrt{s}=19 \mathrm{GeV}$ with $\sigma_{\text {pom }}^{T}=34.92 \mathrm{mb}$ and $B=12.47 \pm 0.10 \mathrm{GeV}^{-2}$. This point has been used [7] as an input in an application of the model of the stochastic vacuum to high-energy scattering, and we now see that it is not consistent (due to a too large value for $B$ ) with the ISR data, as shown in Fig.1. This consideration has influence in the numerical values that are obtained for the QCD parameters. In Fig. 1 we show also the Fermilab CDF values at 1800 $\mathrm{GeV}$, which must be considered as alternative to the values obtained in the E-710 experiment, since they refer to the same energy ; in the analysis presented below we opt for the E-710 values, which fit more naturally in our calculation.

Once the forms of the correlation functions are fixed, the parameters in the model that are fundamentally related to QCD are the weight $\kappa$, the gluon condensate $\left\langle g^{2} F F\right\rangle$ and the correlation length $a$. The hadronic extension parameter $S_{H}$ accounts for the energy dependence of the observables. In this section we show how these quantities can be evaluated using exclusively high-energy scattering data.

To obtain from eqs $(40),(41),(43)$ and (44) a relation between the observables $\sigma^{T}$ and $B$ at a given energy, we eliminate the radius, and write

$$
B-\eta a^{2}=\frac{a^{2}}{\left(<g^{2} F F>a^{4}\right)^{2 \delta / \beta}} \frac{\gamma}{\alpha^{\delta / \beta}}\left(\frac{\sigma^{T}}{a^{2}}\right)^{\delta / \beta} .
$$

The form of eq.(45) is the same as given by eq.(10), with an obvious correspondence of parameters.

To determine the parameters, we first remark that the exponent $\Delta=\delta / \beta$ does not depend on QCD quantities and is almost constant (equal to about $3 / 4$ ) in the region of values of $\kappa$ that are obtained in lattice calculations $(\kappa \approx 3 / 4)$. This tells us that we cannot easily extract a unique value of $\kappa$ from high-energy scattering data only, but tells us also that the power $\Delta$ in eq.(10) must surely be very close to

$$
\delta / \beta=\Delta=3 / 4 .
$$

This is a fortunate result for our analysis, because then in practice we are left with only two free quantities in both energy independent relations (45) and (10). They can be determined using as input the two clean experimental points for $\sigma^{T}$ and $\mathrm{B}$ at 541 and $1800 \mathrm{GeV}$ given in table 1 . We then obtain

$$
\begin{aligned}
& B_{0}=\eta a^{2}=5.38 \mathrm{GeV}^{-2}=0.210 \mathrm{fm}^{2}, \\
& C=0.458 \mathrm{GeV}^{-2} .
\end{aligned}
$$


Fig.1 shows the observables of soft high-energy scattering, with the modification that for the total cross-sections at the ISR energies we show the non-perturbative pomeron-exchange contribution $\sigma_{\text {pom }}^{T}(p p, \bar{p} p)=(21.70 \mathrm{mb}) s^{0.0808}$ instead of the full experimental value. The values of $B$ for the pp system are shown with circles, while the values for $\bar{p} p$ are represented by squares. The solid line is eq.(10), with values for $\Delta, B_{0}$ and $\mathrm{C}$ given above. We see that using the 541 and $1800 \mathrm{GeV}$ data as input, the model gives a very good prediction for the ISR data. It is interesting to observe that the values of $\mathrm{B}$ for the $\mathrm{pp}$ system are closer to our prediction for the non-perturbative slope (the solid line) compared to the $\bar{p} p$ values, which are rather high.

Eq.(45) gives the correspondence between the phenomenological quantities $\Delta, B_{0}, C$ and the parameters of the model and of QCD. Since the model parameters are functions of $\kappa$ only, we can also obtain the QCD parameters as functions of $\kappa$. They are plotted in figs. 5 and 6 . The correlation length is remarkably constant, while the gluon condensate decreases as $\kappa$ increases.

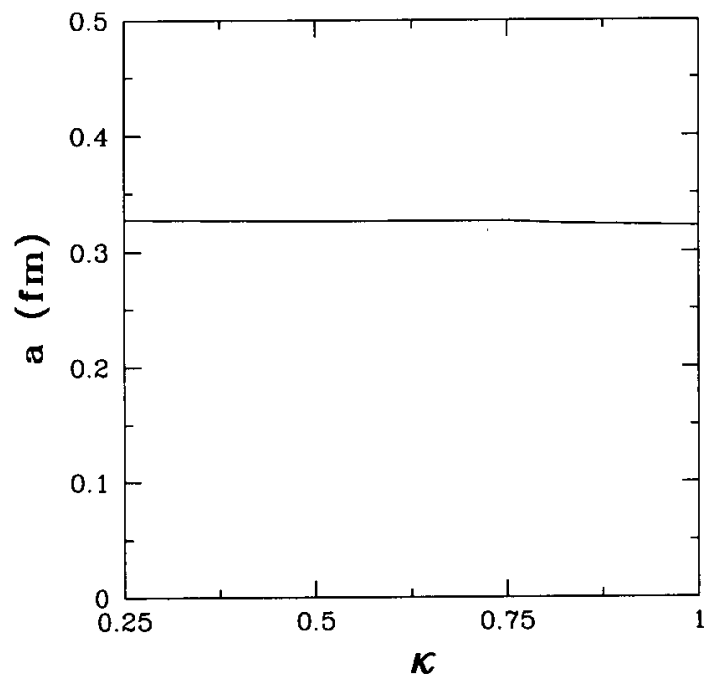

Figure 5. The correlation length as a function of $\kappa$. The values are determined using as input the data at 541 and $1800 \mathrm{GeV}$.

Of course these results are subject to uncertainties. We have adopted an ansatz for the correlation function, which is arbitrary (although numerically it could not be very different). There is some uncertainty also in the determination of the parameters $\alpha, \beta \ldots$ representing the final results of the numerical calculation. On the other hand, the model gives a rather unique prediction for $\Delta=\delta / \beta=3 / 4$ which is well sustained by the data as shown in Fig.1.

To be specific, we borrow from lattice calculation the value $\kappa=3 / 4$, and then use the parameter values obtained for this case. Taking into account the experimental error bars in the input data at 541 and 1800
$\mathrm{GeV}$, we obtain

$$
\begin{gathered}
\kappa=3 / 4, a=0.32 \pm 0.01 \mathrm{fm} \\
\quad<g^{2} F F>a^{4}=18.7 \pm 0.4 \\
<g^{2} F F>=2.7 \pm 0.1 \mathrm{GeV}^{4}
\end{gathered}
$$

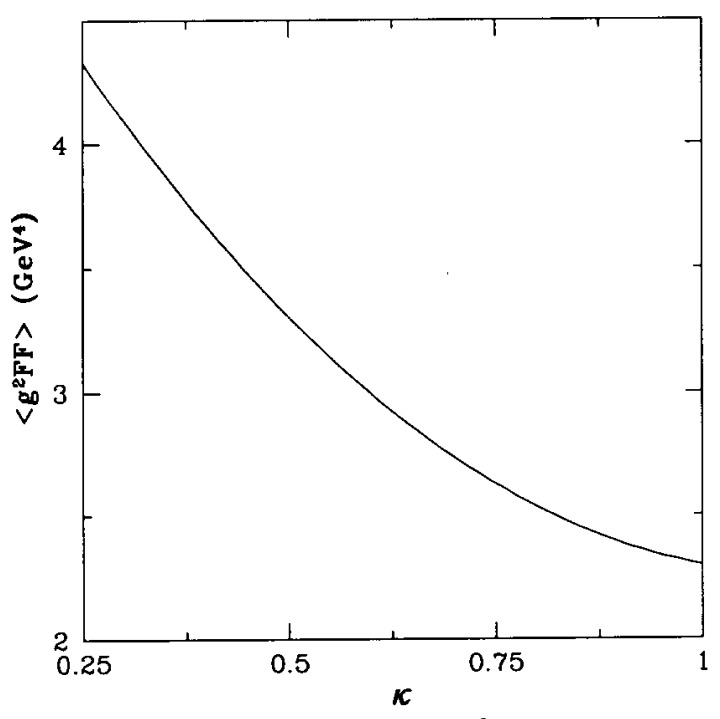

Figure 6. The gluon condensate $\left\langle g^{2} F F\right\rangle$ as a function of $\kappa$, determined using as input the data at 541 and 1800 $\mathrm{GeV}$.

With the value $\kappa=33 / 40$ obtained in more recent lattice results [21] the central values change slightly to

$$
\begin{aligned}
& \kappa=33 / 40, a=0.33 \mathrm{fm}, \\
& <g^{2} F F>a^{4}=19.2 \text {, } \\
& <g^{2} F F>=2.6 \mathrm{GeV}^{4} \text {. }
\end{aligned}
$$

The results of the pure $S U(3)$ lattice gauge calculation by Di Giacomo and Panagopoulos [11] for the correlator $\left\langle F_{\mu \nu}^{C}(x, 0) F_{\rho \sigma}^{D}(0,0)\right\rangle_{A}$ have been fitted [7] with the same correlation function $(22)$ used in the present work. The correlation between the values of $\left\langle g^{2} F F\right\rangle$ and $a$ that was then obtained can be well represented by the empirical expressions

$$
\begin{array}{r}
\Lambda_{L}=\frac{1.1122}{a^{1.310}}, \quad\left\langle g^{2} F F>=\frac{0.01813}{a^{4.656}},\right. \\
<g^{2} F F>a^{4}=0.0172 \sqrt{\Lambda_{L}}
\end{array}
$$

with the lattice parameter $\Lambda_{L}$ in $\mathrm{MeV}, a$ in fm, and $\left\langle g^{2} F F\right\rangle$ in $\mathrm{GeV}^{4}$. This correlation is displayed in Fig.7, where some chosen values of $\Lambda_{L}$ are marked. $\Lambda_{L}$ usually takes values in the range $5 \pm 1.5 \mathrm{MeV}$. The point representing our results of eq.(48) is marked in the same figure. The dashed line represents the relation between the gluon condensate, the correlation length and the string tension obtained in the application of the model of the stochastic vacuum [8] to hadron spectroscopy; for our form of correlator, this relation is given by eq.(25). The curve drawn corresponds to a string tension $\rho=0.16 \mathrm{GeV}^{2}$. 


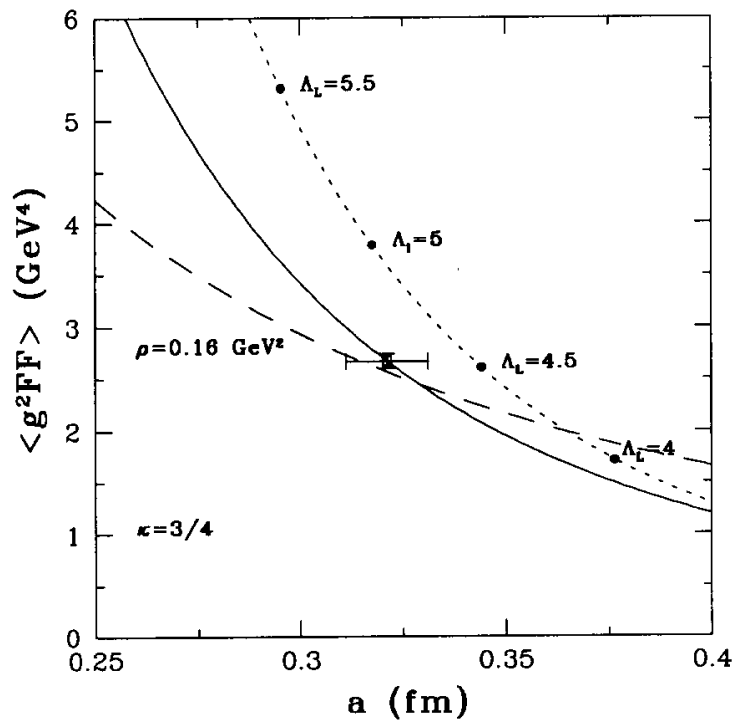

Figure 7. Constraints on the values of $\left\langle g^{2} F F\right\rangle$ and of the correlation length $a$. The solid line is the fit of our correlator to the lattice calculation [11] as given in eq.(50). The dashed line plots eq. (25), with $\rho=0.16 \mathrm{GeV}^{2}$. The cross centered at $a=0.32 \mathrm{fm},\left\langle g^{2} F F>=2.7 \mathrm{GeV}^{4}\right.$ shows the result of our calculation given in eq.(48).

As can be seen from the figure, the constraints from these three independent sources of information are simultaneously satisfied, providing a consistent picture of soft high-energy $p p$ and $\bar{p} p$ scattering. The (pure gauge) gluon condensate is well compatible with the expected value. The lattice parameter $\Lambda_{L}$ and the string tension $\rho$ are also in their acceptable ranges. As we describe below, the resulting proton size parameter $S_{p}$ takes values quite close to the electromagnetic radius [22].

In our model the increase of the observables with the energy is due to a slow energy dependence of the hadronic radii. An explicit relation is obtained if we bring into eqs.(40) and (43) a parameterization for the energy dependence of the total cross-sections, such as the Donnachie-Landshoff [3] form. In this case we obtain for the proton radius

$$
S_{p}(s)=\frac{a}{\alpha^{1 / \beta}}\left(\frac{21.7 \mathrm{mb}}{a^{2}}\right)^{1 / \beta} \frac{s^{0.0808 / \beta}}{\left(\left\langle g^{2} F F\right\rangle a^{4}\right)^{2 / \beta}} .
$$

The energy dependence, given by a power $0.0808 / \beta$ of $\mathrm{s}$ is very slow, and the values obtained for $S_{p}$ are in the region of the proton electromagnetic radius [22], which is $R_{p}=0.862 \pm 0.012 \mathrm{fm}$. However, use of the DonnachieLandshoff parameterization for the total cross-sections is not appropriate at very high energies. Using eqs. (40) and (43) and directly the data at 541 and $1800 \mathrm{GeV}$, we obtain the values for the proton radius that are shown in Fig.8, where a log scale is used for $\sqrt{s}$. It is remarkable that we have an almost linear dependence, which can be represented by

$$
S_{p}(s)=0.671+0.057 \log \sqrt{s}(\mathrm{fm}),
$$

with $\sqrt{s}$ in $\mathrm{GeV}$. With this form for the radius, which is shown in dashed line in Fig.8, the cross-sections evaluated at very high energies rise with a term $\log ^{\beta} \sqrt{s}$, and are smaller than predicted by the power dependence of Donnachie-Landshoff. However, since $\beta \approx 2.8$, they still violate the bound $\log ^{2} \sqrt{s}$. This may be corrected using a power $2 / \beta$ instead of 1 in the parameterization for $S_{p}(s)$, and we then obtain

$$
S_{p}(s)=0.572+0.123[\log \sqrt{s}]^{0.72}(\mathrm{fm}) .
$$

This form is shown in solid line in Fig.(8). Clearly it gives a good representation for the existing data. At $14 \mathrm{TeV}$, which is the expected energy in the future LHC experiments, we obtain $S_{p}(14 \mathrm{TeV})=1.19 \mathrm{fm}=$ $1.38 R_{p}=3.7 a$ and the model predictions for the observables are $\sigma^{T}=92 \mathrm{mb}$ and $\mathrm{B}=19.6 \mathrm{GeV}^{-2}$. The dashed line representing eq.(52) leads at the same LHC energy to $\sigma^{T}=97 \mathrm{mb}$ and $\mathrm{B}=20.1 \mathrm{GeV}^{-2}$, while the Donnachie-Landshoff formula leads to the still higher value $\sigma^{T}=101.5 \mathrm{mb}$.

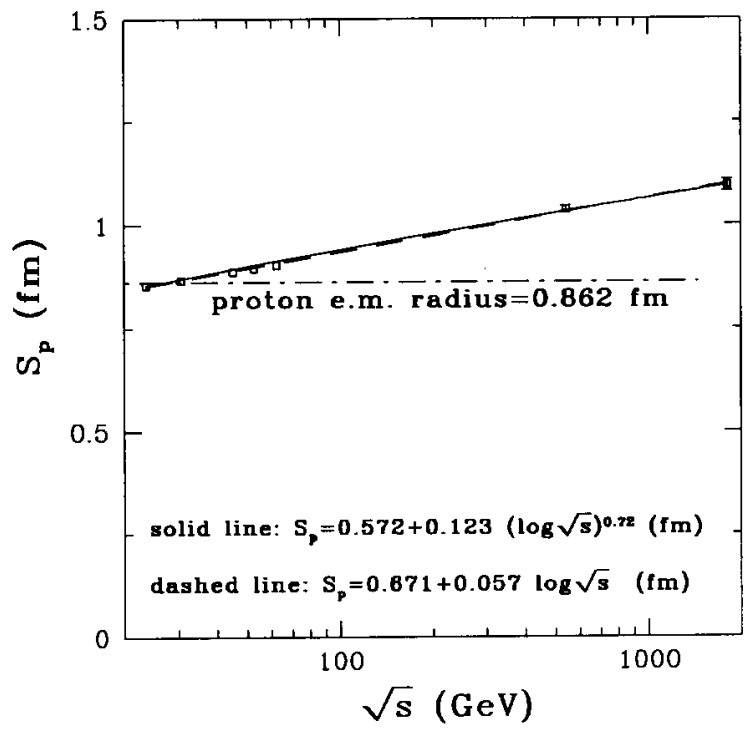

Figure 8. Energy dependence of the proton radius. The marked points are obtained from the total cross-section data (at the ISR energies the total cross-sections are represented by the pomeron exchange contributions). The two representations for the radius dependence are indistinguishable with the present data, but give quite different predictions for the cross-section values at the LHC energies.

The non-perturbative QCD contributions to soft high-energy scattering are expected to be dominant in the forward direction, thus determining the total crosssection (through the optical theorem) and the forward slope parameter. The model, as it is presented in this paper, leads to a negative curvature for the slope $\mathrm{B}(\mathrm{t})$, which decreases as $|t|$ increases, as shown in Fig.5. The data however shows an almost zero curvature of the peak, so that above some value of $|t|$ the model leads to too high values of the differential cross-section. This is illustrated in Fig.9, where the experimental data [13] 
at 541,546 and $1800 \mathrm{GeV}$ are shown, together with the results of the model, without any free parameter (the gluon condensate, the correlation length and the hadronic radius have been uniquely fixed by the inputs of $\sigma^{T}$ and $\mathrm{B}$ at 541 and $1800 \mathrm{GeV}$ ).
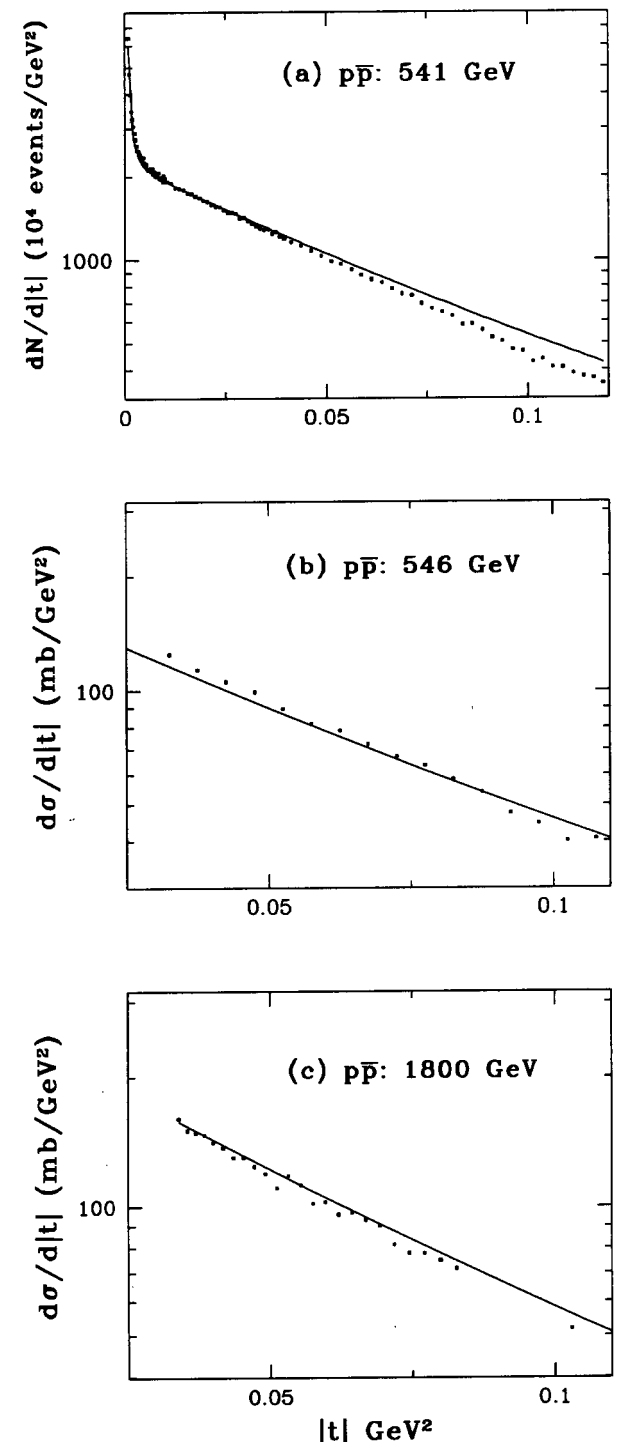

Figure 9. Elastic differential cross-sections at high energies [13]. The solid lines represent the calculations with the model of the stochastic vacuum described in the present work, without free parameters. The systematic deviations occurring for $|t|$ larger about $30 \times 10^{-3} \mathrm{GeV}^{2}$ arise from the long range behavior of the profile function $\mathrm{J}(\mathrm{b} / \mathrm{a})$.

\section{Final remarks}

We have explained the calculation of soft high-energy scattering, including the two tensor forms in the correlator, and thus taking into account the two independent correlation functions. We mentioned the influence of the weight parameter $\kappa$ that measures the ratio between the two contributions, giving the general results that allow the determination of the observable quantities and QCD parameters in terms of this weight. We show that there are little changes in the final results, when $\kappa$ varies in the ranges suggested by lattice determinations.

The model of the stochastic vacuum describes the most important data on total cross-section and slope parameter for the pp and $\bar{p}$ p systems, extended from $\sqrt{s} \approx 20$ to $1800 \mathrm{GeV}$, giving a unified and consistent description of these data in terms of fundamental quantities. The non-perturbative QCD parameters determining the observables are the gluon condensate and the correlation length of the vacuum field fluctuations. The third quantity entering the calculations is the transverse hadron size, which has a magnitude close to the electromagnetic radius, and whose variation accounts for the energy dependence of the observables.

The model allows a very convenient factorization between the QCD and hadronic sectors. Elimination of the hadron size parameter between the expressions for the two observables at a given energy yields a parameter-free and energy independent relation between the total cross-section and the slope of the elastic cross-section which agrees very well with experiment. Starting from two experimental energies as input, this expression gives a prediction of the remaining data, and leads to a determination of the correlation length and the gluon condensate from high-energy data alone. The results obtained are in good agreement with the correlations between the two QCD parameters obtained in the lattice calculations and in the application of the stochastic vacuum model to hadronic spectroscopy.

In the expansion of the exponential with functional integrations, the present calculation is restricted to the lowest order non-vanishing contribution, which is quadratic in the gluonic correlator, and we may conclude from our results that this is justified for the evaluations of total cross-section and slope parameter. The resulting amplitude is purely imaginary, and the $\rho$ parameter (the ratio of the real to the imaginary parts of the elastic scattering amplitude) can only be described if we go one further order in the contributions to the correlator. Also the factorization in eq.(16), implied by the assumption of a Gaussian process, is important in the present formulation of the model, and possibly has consequences for the phenomenological analysis. Recent developments of the model [23] give more accurate determination of the profile function as a function of the impact parameter, without requiring the assumption of small values the function $\chi$. There is important improvement in the predicted t-dependence of the differential cross-section. 


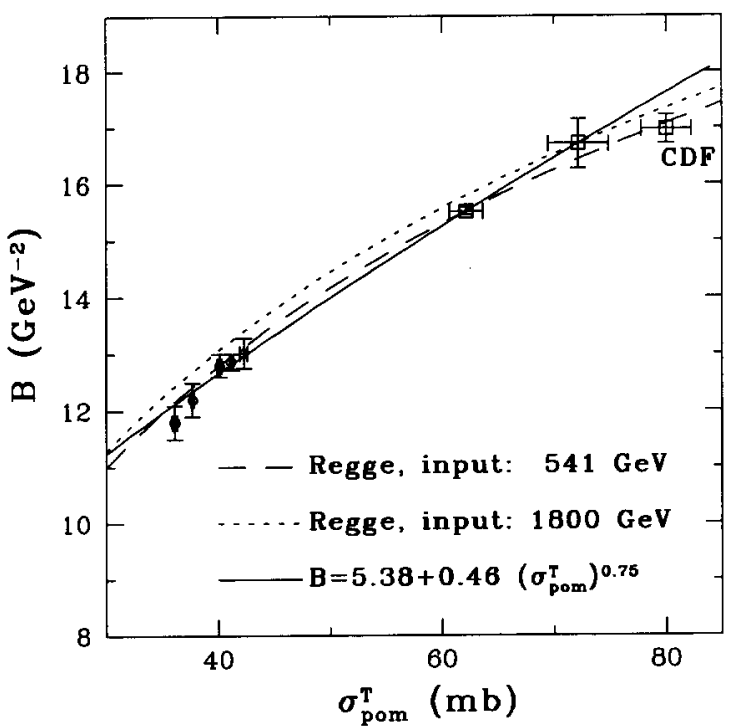

Figure 10. Relation between observables of high-energy scattering obtained in our calculations compared to the relation obtained from a Regge amplitude according to eq.(54). The dashed line uses as input for the Regge formula the $\sqrt{s}=541 \mathrm{GeV}$ data and passes close to the CDF point at $1800 \mathrm{GeV}$. The dotted line uses as input the values of the E-710 experiment at $\sqrt{s}=1800 \mathrm{GeV}$.

It is interesting to compare the results of the model of the stochastic vacuum with Regge phenomenology. In Fig. 10 we plot the relation between the observables given by

$$
\sigma_{\text {Regge }}^{T}=\sigma_{0}^{T} e^{0.1616\left(B-B_{0}\right)},
$$

obtained from a Regge amplitude using the slope of the pomeron trajectory $\alpha^{\prime}(0)_{\text {pom }}=0.25 \mathrm{GeV}^{-2}$. This relation requires an input pair $\sigma_{0}^{T}, B_{0}$ at a chosen energy. The dashed line uses as input the $\sqrt{s}=541 \mathrm{GeV}$ data $\sigma_{0}^{T}=62.20 \mathrm{mb}$, and $B_{0}=15.52 \mathrm{GeV}^{-2}$. It is interesting to observe the tendency of this line to pass close to the CDF experimental point, instead of the E710 point. The dotted line uses as input the values of the E-710 experiment at $\sqrt{s}=1800 \mathrm{GeV}$ and shows a deviation at $541 \mathrm{GeV}$. This is rather intriguing, as it implies that eq.(54) favors the CDF experimental results at $1800 \mathrm{GeV}$.

\section{Acknowledgments}

We are grateful to H.G. Dosch for the kind and fruitful collaboration extended along several years, and acknowledge the participation of Flávio Pereira in the calculations with the model of the stochastic vacuum that are here reported.

\section{References}

[1] Review Articles: L.L. Jenkovszky, Fort. Phys. 34, 791 (1986); M.M. Block and R.N. Cahn, Rev. Mod. Phys. 57, 563 (1985).
[2] P.D.B. Collins, An Introduction to Regge Theory and High Energy Physics - (Cambridge University Press, 1977).

[3] A. Donnachie and P.V. Landshoff, Phys. Lett. B 296, 227 (1992).

[4] M. Froissart, Phys. Rev. 123, 1053 (1961); A. Martin, Phys. Rev. 129, 1432 (1963).

[5] P.V. Landshoff and O. Nachtmann, Z. Phys. C 35, 405 (1987).

[6] O. Nachtmann, Ann. Phys. 209 (1991) 436.

[7] H.G. Dosch, E. Ferreira and A. Krämer, Phys. Lett. B 289, 153 (1992); Phys. Lett. B 318, 197 (1993); Phys. Rev. D 50, 1992 (1994); E. Ferreira and F. Pereira, Phys. Rev. D55, 130 (1997);

[8] H.G. Dosch, Phys. Lett. B 190, 177 (1987).

[9] H.G. Dosch and Yu.A. Simonov, Phys. Lett. B 205, 339 (1988).

[10] H.G. Dosch, Lectures on Hadron Physics, Ed. E. Ferreira, World Scientific (1990) p.40.

[11] A. Di Giacomo and H. Panagopoulos, Phys. Lett. B 285, 133 (1992).

[12] B. Povh and J. Hüfner, Phys. Rev. Lett. 58, 1612 (1987), Phys. Lett. B 215, 772 (1988) and B 245, 653 (1990); Phys. Rev. D 46, 990 (1992) 990; C. Bourrely, J. Soffer and T.T. Wu, Nucl. Phys. B 247, 15 (1984)15; Phys. Rev. Lett. 54, 757 (1985); Phys. Lett. B 196, 237 (1987)7; J. Dias de Deus and P. Kroll, Nuovo Cimento A 37, 67 (1977) 67; Acta Phys. Pol. B 9, 157 (1978); J. Phys. G 9, L81 (1983); P. Kroll, Z. Phys. C 15, 67 (1982); T.T. Chou and C.N. Yang, Phys. Rev. 170, 1591 (1968) and D 19, 3268 (1979); Phys. Lett. B 128, 457 (1983) and B 244, 113 (1990); E. Levin and M.G. Ryskin, Sov. J. Nucl. Phys. 34, 619 (1981).

[13] Main references to the data. (a) N. Amos et al, Nucl. Phys. B 262, 689 (1985); (b) R. Castaldi and G. Sanguinetti, Ann. Rev. Nucl. Part. Sci. 35, 351 (1985); (c) C. Augier et al, Phys. Lett. B 316, 448 (1993); (d) M. Bozzo et al, Phys. Lett. B 147, 392 (1984); M. Bozzo et al, Phys. Lett. B 147, 385 (1984); (e) N. Amos et al, Phys. Lett. B 247, 127 (1990); Phys. Rev. Lett. 68, 2433 (1992); Fermilab-FN-562 [E-710] (1991).

[14] Data Compilations, Particle Data Group, Review of Particle Properties, Phys. Rev. D 50 (1994) number 3, part I; Review Articles, L.L. Jenkovszky, Fort. Phys. 34, 791 (1986); M.M. Block and R.N. Cahn, Rev. Mod. Phys. 57, 563 (1985).

[15] G. Alner, Zeit. Phys. C 32, 153 (1986).

[16] F. Abe et al. Phys. Rev. D 50, 5550 (1994); Phys. Rev. D 50, 5518 (1994).

[17] J.B. Burq et al, Nucl. Phys. B 217, 285 (1983).

[18] M.A. Shifman, A.I. Vainshtein and V.I. Zakharov, Nucl. Phys. B 147, 385, 448 and 519 (1979).

[19] N.G.van Kampen, Physica 74, 215, 239 (1974) and Phys. Rep. C 24, 172 (1976); G.C. Hegerfeldt and H. Schulze, J. Stat. Phys. 51, 691 (1988).

[20] M. Schiestl and H.G. Dosch, Phys. Lett. B 209, 85 (1988); Y.A. Simonov, Nucl. Phys. B 234, 67 (1989). 
[21] A. Di Giacomo, E. Maggiolaro and H. Panagopoulos, hep-lat/9603017, March 1996.

[22] Proton radius: G.G. Simon et al. Z. Naturforschung A
35, 1 (1980).

[23] E.R. Berger and O. Nachtmann, Eur. Phys. J. C 7, 459 (1999); hep-ph/9808320. 